\title{
Conocimiento de la logopedia. Un estudio descriptivo
}

Speech therapy knowledge. A descriptive study

Elena Rubio-Iglesias Fernández-Vegue

Graduada en Logopedia. Facultad de Ciencias de la Salud. Universidad de Castilla-La Mancha. Talavera de la Reina, Toledo, España helenarubioiglesias@hotmail.com

Manuela Martínez Lorca

Departamento de Psicología. Facultad de Ciencias de la Salud. Universidad de Castilla-La Mancha. Talavera de la Reina,Toledo, España manuela.martinez@uclm.es

Alberto Martínez Lorca

Servicio de Medicina Nuclear del Hospital Universitario Ramón y Cajal, Madrid Departamento de Enfermería, Fisioterapia y Terapia Ocupacional. Facultad de Ciencias de la Salud. Universidad de Castilla-La Mancha. Talavera de la Reina, Toledo, España alberto.mlorca@uclm.es Juan José Criado Álvarez

Departamento de Ciencias Médicas. Facultad de Ciencias de la Salud. Universidad de Castilla-La Mancha. Talavera de la Reina, Toledo, España Instituto de Ciencias de la Salud de Castilla-La Mancha. Consejería de Sanidad. Talavera de la Reina, Toledo, España jjcriado@secam.jccm.es

Fecha recepción: 21/01/2020

Páginas 1-13

Fecha aceptación: 29/05/2020

\section{Resumen.}

La Logopedia es una disciplina del ámbito sanitario que realiza la evaluación, el diagnóstico, la prevención, la intervención y el estudio de dificultades, patologías y alteraciones en el lenguaje (oral y escrito), habla, voz, audición y funciones orales no verbales. Actualmente, se encuentran escasos estudios que investiguen el conocimiento que tiene la población española sobre la logopedia. Por tanto, el objetivo de este trabajo es conocer qué conocimiento se tiene sobre la logopedia. Se ha realizado un estudio descriptivo mediante un cuestionario elaborado "ad hoc" en formato on-line de 34 preguntas, con el que se analizan aspectos como el contacto que tienes los participantes con la logopedia, los campos de actuación de un logopeda, las alteraciones que trata y la valoración de la logopedia. La muestra fue de 299 personas con una sub-muestra de 64 porque se excluyeron las que estudian o estudiaban logopedia, que son logopedas, que han asistido al logopeda o bien que conocen a alguien relacionado con la logopedia. Se concluyó que la logopedia sí es conocida por la población, aunque hay cierta desinformación sobre algunas áreas. Se recomienda realizar investigaciones a mayor escala, para alcanzar unos resultados más precisos.

Palabras clave: logopedia, cuestionario "ad hoc", conocimiento, imagen, profesión 


\begin{abstract}
.
The Speech-Language Therapy is a health discipline that do assessment, diagnosis, prevention, treatment and study of language, speech, voice, audiology and non-verbal oral functions disorder, pathologies and disturbances, from babies to the oldest. Currently there are few studies where the knowledge of the Spanish population about speech therapy is investigated. Therefore, this research aims to know what knowledge they have about the Speech-Language Therapy. A study was carried out through an online questionnaire of 34 questions, which analyzed aspects such as the image of the speech therapist, the professional work of the speech-language therapist and the assessment of speech therapy. The sample was 299 people, with a sub-sample of 64 because were put away who study or studied Speech-Language Therapy, who are speech-language pathologist, who have attended the speech-language pathologist or who know someone related to Speech-Language Therapy. Afterwards, the results were analyzed and it was concluded that Speech-Language Therapy is known by the population although there is some misinformation about some areas. It recommended research on a larger scale, to achieve more accurate results.
\end{abstract}

Keywords: speech therapy, questionnaire, knowledge, image, profession

\title{
1.-Introdución.
}

La Logopedia en España es una disciplina reciente y joven si la comparamos con otras, como la medicina, enfermería o psicología. Así en los años 60 se funda la primera asociación de logopedas, llamada Asociación Española de Logopedia y Fonología (AELF), actualmente AELFA-IF y en 1998 se crea el primer Colegio de Logopedas en España, siendo el Colegio de Logopedas de Cataluña tal y como se recoge en la ley 2/1998, de 19 de febrero, de creación del Colegio de Logopedas da Cataluña.

Con respecto a los estudios de Logopedia, no fue hasta 1985 cuando se introdujeron los primeros estudios de Logopedia en la universidad pública, concretamente en las Universidades Complutense y Autónoma de Madrid, y en 1992 se aprueba la Diplomatura de Logopedia en el Real Decreto 1419/1991, de 30 de agosto, que fue instaurándose en distintas universidades de España como Valladolid, Salamanca, Oviedo, A Coruña, Málaga, CastillaLa Mancha. Más tarde en el 2007 se creó el Grado de Logopedia, el cual está disponible, tanto en universidades públicas como privadas, en la mayoría de las comunidades autónomas, como son Galicia, Cataluña, Castilla-La Macha, Comunidad Valenciana, Comunidad de Madrid, Andalucía, Las Islas Canarias, la Región de Murcia, Asturias y Castilla y León.

Por tanto, se puede decir que la Logopedia es una disciplina relativamente reciente, donde la población general conoce esta disciplina, aunque tiene lagunas en algunos aspectos (López, 2017). Por ello, se han realizado campañas con el propósito de visualizar la Logopedia, como la dirigida en 2016 por el Consejo General de Colegios de Logopedas (CGCL), titulada ¿Qué es la logopedia para ti? o en 2018 por el Colegio de Logopedas de Cataluña, Un logopeda hace mucho más de lo que piensas. 
Sin embargo, en otros países sí se han realizado trabajos de investigación sobre el conocimiento, funciones, objetivos y campos de actuación de la Logopedia y del logopeda (Breadner, Warr-Leeper y Husband, 1987; Pimentel, Lopes-Herrera y Duarte, 2010; Santo, Franco, Arakawa, Xavier, Bastos y Caldana, 2016; Shin Ying Chu, Keng Ping Tang, Grace McConnell, Hanif Farhan Mohd Rasdi y Man-Ching Yuen, 2019).

\section{2.-Metodo.}

\section{1.-Objetivos e hipótesis.}

El objetivo de este trabajo es estudiar el conocimiento que las personas tienen sobre la logopedia a partir de una encuesta en una muestra española. Nuestra hipótesis es que los participantes tienen conocimiento de la logopedia.

\section{2.-Metodología seguida en la investigación.}

El presente trabajo es estudio epidemiológico descriptivo u observacional.

\section{3.-Participantes.}

La muestra ha sido de 299 personas residentes en España, de las cuales el 70,2\% ( $N=210)$ son mujeres y el $29,8 \%$ ( $N=89$ ) son hombres, con edades comprendidas entre 20 y 85 años (media de 40 años y una desviación típica de 15,32 años). De dicha muestra fueron excluidos 235 personas que estudian o estudiaban logopedia, que son logopedas, que han asistido al logopeda o que conocen a alguien relacionado con la logopedia. Estos criterios de exclusión tienen como objetivo obtener unos resultados más exhaustivos. Por tanto, la muestra final es de 64 personas, de las cuales el 59,37\% ( $N=38)$ son mujeres y el $40,63 \%(N=26)$ son hombres, con edades comprendidas entre 20 años y 70 años (media de 41,56 años y una desviación típica de 13,38 años). Las características de la muestra original (M1) ( $N=299)$ y reducida (M2) $(\mathrm{N}=64)$ según la Comunidad Autónoma, nivel de estudios, estudios que están cursando y profesión se resumen en la Tabla 1.

Tabla 1.-Distribución de la muestra por Comunidades Autónomas, nivel de estudios, estudios que están cursando y profesión.

\begin{tabular}{lcccc}
\hline & $\mathbf{N}=\mathbf{2 9 9}$ & \multicolumn{3}{c}{$\mathbf{N = 6 4}$} \\
\hline Comunidades Autónomas & $\mathbf{N}^{\mathbf{0}}$ de personas & \% & $\begin{array}{c}\mathbf{N}^{\mathbf{0}} \text { de } \\
\text { personas }\end{array}$ \\
\hline Castilla-La Mancha & 158 & 52,8 & 28 & $\%$ \\
Andalucía & 53 & 17,7 & 19 & 29,8 \\
Comunidad de Madrid & 46 & 15,4 & 9 & 14 \\
Extremadura & 23 & 7,7 & 4 & 6,3 \\
Cataluña & 7 & 2,3 & 1 & 1,6 \\
Aragón & 3 & 1 & -- & - \\
Cantabria & 2 & 0,7 & -- & - \\
Comunidad Valenciana & 2 & 0,7 & -- & - \\
La Rioja & 2 & 0,7 & 1 & 1,6 \\
Castilla y León & 1 & 0,3 & 1 & 1,6 \\
\hline
\end{tabular}




\begin{tabular}{|c|c|c|c|c|c|}
\hline Galicia & 1 & 0,3 & -- & & -- \\
\hline País Vasco & 1 & 0,3 & 1 & & 1,6 \\
\hline Nivel de estudios & $\mathrm{N}^{0}$ de personas & $\%$ & $\begin{array}{c}\mathrm{N}^{0} \text { de } \\
\text { personas }\end{array}$ & & $\%$ \\
\hline Educación Primaria & 16 & 5,4 & 3 & & 4,7 \\
\hline EducaciónSecundaria Obligatoria & 17 & 5,7 & 5 & & 7,8 \\
\hline Bachillerato & 52 & 17,4 & 21 & & 32,8 \\
\hline Ciclo formativo & 26 & 8,7 & 6 & & 9,4 \\
\hline Título Universitario & 153 & 51,2 & 26 & & 40,6 \\
\hline Estudios de posgrado & 35 & 11,7 & 3 & & 4,7 \\
\hline Estudian & $\mathrm{N}^{\circ}$ de personas & $\%$ & $\begin{array}{c}\mathrm{N}^{0} \mathrm{de} \\
\text { personas }\end{array}$ & & $\%$ \\
\hline No & 194 & 64,9 & 49 & & 76,6 \\
\hline Sí & 105 & 35,1 & 15 & & 23,4 \\
\hline Ciencias sociales & 27 & 9 & 6 & & 9,4 \\
\hline Ciencias de la salud & 25 & 8,4 & 3 & & 4,7 \\
\hline Ciencias básicas & 15 & 5,01 & 3 & & 4,7 \\
\hline Idiomas & 14 & 4,7 & 2 & & 3,1 \\
\hline Oposiciones & 13 & 4,3 & 1 & & 1,6 \\
\hline $\begin{array}{l}\text { Cursos de actualización } 0 \\
\text { formación profesional }\end{array}$ & 10 & 3,3 & -- & & -- \\
\hline Profesión & $\mathrm{N}^{0}$ de personas & $\%$ & $\begin{array}{c}\mathrm{N}^{0} \mathrm{de} \\
\text { personas }\end{array}$ & & $\%$ \\
\hline No & 97 & 32,4 & 19 & & 29,7 \\
\hline Sí & 202 & 67,5 & 45 & & 70,3 \\
\hline Sector primario & 2 & 0,7 & 1 & & 1,6 \\
\hline Sector secundario & 7 & 2,3 & 2 & & 3,1 \\
\hline Sector terciario & 193 & 64,5 & 42 & & 65,6 \\
\hline Docente & 44 & & 22,8 & 4 & 6,3 \\
\hline Teleoperador & 20 & & 9 & 3 & 4,7 \\
\hline Administrativo & 17 & & 8,8 & 7 & 10,9 \\
\hline Enfermera & 8 & & 4,1 & 2 & 3,1 \\
\hline
\end{tabular}

Fuente: elaboración propia.

\section{4.-Instrumento.}

Para llevar a cabo este trabajo se utilizó un cuestionario elaborado "ad hoc" teniendo en cuenta los trabajos sobre el conocimiento, la perspectiva pública de la logopedia, las alteraciones y las dificultades que trata un logopeda (Breader et al., 1987; Pimentel et al., 2010; Santo et al., 2016; Shin Ying Chu et al., 2019).

El cuestionario elaborado "ad hoc" consta de 34 preguntas, de las cuales 5 son preguntas abiertas y 30 preguntas cerradas, algunas dicotómicas y otras de respuesta múltiple.

Estas preguntas están divididas en cuatro categorías. La primera, para conocer los datos demográficos con 8 preguntas; la segunda sobre el contacto que han tenido los participantes con la logopedia, desde la pregunta 9 hasta la 21 ; la tercera categoría, de la pregunta 22 hasta la 29, sobre la labor profesional y las alteraciones que trata el logopeda; y la cuarta sobre la valoración de la logopedia, con las últimas 5 preguntas.

La creación de dicho cuestionario se hizo a través de Formularios de Google. Es una aplicación de Google donde se recopila y organiza todo tipo de información, se recibe las respuestas de manera instantánea, se puede elegir la manera de realizar las preguntas, (listas desplegables, opción múltiple, casillas de verificación, respuesta corta, escala lineal, etc.) y 
tiene un diseño que se puede modificar y contestar en cualquier aparato electrónico. Una vez recopiladas las respuestas, las organiza y analiza a través de gráficos y guarda las respuestas en una hoja de cálculo en línea. Además, es una herramienta totalmente gratuita. Si bien, el encuestado tiene que tener servicio de Internet ya que no tiene una función off-line.

\section{5.-Procedimiento.}

Tras haber creado el cuestionario "ad hoc", este fue evaluado mediante un papel de expertos compuesto por logopedas, personas que han acudido al logopeda y profesorado universitario del Grado de Logopedia. Después de realizar cambios estructurales, formales, mejorar la comprensión de algunos conceptos y añadir o quitar algunas opciones, se llevó a cabo un estudio piloto, con un grupo de 10 personas ajeno a la Logopedia, para confirmar que la redacción final del cuestionario "ad hoc" tras los cambios realizados era comprendido por personas no vinculadas con el ámbito logopédico.

Finalmente, se publicó la versión definitiva del cuestionario "ad hoc". de manera on-line en Formularios de Google. Antes de que los participantes accedieran al cuestionario, tenían que rellenar el consentimiento informado. En este se mostraba el objetivo del estudio junto a la ley de protección de datos teniendo en cuenta la ley orgánica 3/2018, de Protección de Datos Personales y garantía de los derechos digitales.

Para su difusión se utilizaron diferentes plataformas y aplicaciones como el correo electrónico, Facebook y Whatsapp. Este cuestionario estuvo disponible en la plataforma on-line desde el día 24 de febrero del 2018 hasta el 3 de abril del 2018, siendo este el periodo de tiempo durante el cual fue recogida la muestra. Finalizada esta, se comenzó con el análisis de los datos mediante el programa informático IBM ${ }^{\circ}$ SPSS $\circledast$ Statistics 22.0. Para el análisis estadístico se utilizaron los estadísticos para la distribución de frecuencias y descriptivos (principalmente, medias y desviaciones típicas).

\section{3.-Resultados.}

\section{1.-Contacto con la logopedia.}

Los resultados de este apartado pueden verse en la siguiente tabla (Tabla 2). Sin embargo, algunos datos relevantes indican que una amplia mayoría dice saber lo que es un logopeda $(89,1 \%)$, al igual que saben que Logopedia es un Grado Universitario (82,8\%).

Entre las patológicas más frecuentes por las que se solicita cita al logopeda, destacan las dificultades en el habla y las dificultades en la voz. Desconociéndose para el $58,1 \%$ el coste de la consulta y un $25,8 \%$ considero que fue económico, sin embargo, para un porcentaje elevado ( $83,9 \%$ de la muestra total frente al $68,8 \%$ de la muestra reducida) sabe que la seguridad social cubre este servicio.

A la pregunta si alguna vez han recibido información acerca de la logopedia, se observa cómo el $57,8 \%$ de las personas de M2 sí han recibido información con respecto al 70,9\% de M1 y sobre todo a través de charlas informativas, las redes sociales y otros medios. 
Tabla 2.-Distribución de las respuestas dadas a las preguntas 9 a la 21.

\begin{tabular}{|c|c|c|c|c|}
\hline \multirow[b]{2}{*}{ 9. Sabe lo que es un logopeda } & \multicolumn{2}{|c|}{$\mathrm{N}=299$} & \multicolumn{2}{|c|}{$\mathrm{N}=64$} \\
\hline & $\begin{array}{c}\mathrm{N}^{0} \text { de } \\
\text { personas }\end{array}$ & $\%$ & $\begin{array}{c}\mathrm{N}^{0} \text { de } \\
\text { personas }\end{array}$ & $\%$ \\
\hline Sí & 292 & 97,7 & 57 & 89,1 \\
\hline No & 7 & 2,3 & 7 & 10,9 \\
\hline \multicolumn{5}{|l|}{ 10. Logopedia es un Grado Universitario } \\
\hline Sí & 269 & 90 & 53 & 82,8 \\
\hline No & 30 & 10 & 11 & 17,2 \\
\hline \multicolumn{5}{|l|}{ 11. Estudia o ha estudiado Logopedia } \\
\hline Sí & 30 & 10 & 0 & 0 \\
\hline No & 269 & 90 & 64 & 100 \\
\hline \multicolumn{5}{|l|}{$\begin{array}{l}\text { 12. Conoce a alguien que estudia o ha } \\
\text { estudiado Logopedia }\end{array}$} \\
\hline Sí & 231 & 77,3 & 0 & 0 \\
\hline No & 68 & 22,7 & 64 & 100 \\
\hline \multicolumn{5}{|l|}{ 13. Ha asistido al logopeda } \\
\hline Sí & 31 & 10,4 & 0 & 0 \\
\hline \multicolumn{5}{|l|}{ 14. Motivo } \\
\hline Trastornos del habla (dislalia o disfemia) & 16 & 51,6 & -- & -- \\
\hline Problemas en la voz & 8 & 25,8 & -- & -- \\
\hline Dificultades en el lenguaje & 4 & 12,9 & -- & - \\
\hline Como acompañante a un familiar & 3 & 9,8 & -- & -- \\
\hline \multicolumn{5}{|l|}{ 15. Lugar } \\
\hline Colegio & 10 & 32,3 & -- & -- \\
\hline Consulta & 4 & 12,9 & -- & -- \\
\hline Seguridad Social & 2 & 6,5 & -- & -- \\
\hline A domicilio & 3 & 9,6 & -- & -- \\
\hline Su ciudad de residencia & 12 & 38,7 & -- & -- \\
\hline \multicolumn{5}{|l|}{ 16. Coste } \\
\hline Económico & 8 & 25,8 & -- & -- \\
\hline Costoso & 5 & 16,1 & -- & -- \\
\hline No sabe/No contesta & 18 & 58,1 & -- & -- \\
\hline No & 268 & 89,6 & 64 & 100 \\
\hline \multicolumn{5}{|l|}{$\begin{array}{l}\text { 17. Conoce a alguien que haya asistido } \\
\text { al logopeda }\end{array}$} \\
\hline Sí & 250 & 83,6 & -- & -- \\
\hline No & 49 & 16,4 & 64 & 100 \\
\hline \multicolumn{5}{|l|}{$\begin{array}{l}\text { 18. Recibido o visto información sobre } \\
\text { la Logopedia }\end{array}$} \\
\hline Sí & 212 & 70,9 & 37 & 57,8 \\
\hline No & 87 & 29,1 & 27 & 42,2 \\
\hline \multicolumn{5}{|l|}{ 19. A través de que medio } \\
\hline Redes sociales & 40 & 18,9 & 7 & 18,9 \\
\hline Anuncios & 5 & 2,4 & 1 & 2,7 \\
\hline Charlas informativas & 39 & 18,4 & 6 & 16,2 \\
\hline Otros medios & 151 & 71,2 & 27 & 73 \\
\hline Carteles y/o folletos & 22 & 10,4 & 4 & 10,8 \\
\hline $\begin{array}{l}\text { 20. Existe un centro logopédico en el } \\
\text { lugar donde vive }\end{array}$ & \multicolumn{2}{|c|}{$\begin{array}{l}\mathrm{N}^{0} \text { de personas } \\
\%\end{array}$} & $\begin{array}{c}\mathrm{N}^{0} \text { de } \\
\text { personas }\end{array}$ & $\%$ \\
\hline Sí & 190 & 63,5 & 36 & 56,3 \\
\hline No & 109 & 36,5 & 28 & 43,7 \\
\hline $\begin{array}{l}\text { 21. Puede acceder al logopeda a través } \\
\text { de la Seguridad Social }\end{array}$ & & & & \\
\hline
\end{tabular}




\begin{tabular}{lcccc}
\hline Sí & 251 & 83,9 & 44 & 68,8 \\
No & 48 & 16,1 & 20 & 31,3 \\
\hline
\end{tabular}

Fuente: elaboración propia.

3.2.-Campo de actuación de un logopeda y alteraciones que trata.

En relación con los resultados obtenidos en la tercera categoría, estos se muestran en la Tabla 3, sin embargo, se destaca como los bebés y los ancianos en ambas muestras presentan menores porcentajes frente a niños, adolescentes y adultos. También es reseñable los distintos lugares en los que trabaja el logopeda desde la clínica privada hasta la asociación. Para el 73,9\% (muestra pequeña) y para el $71,9 \%$ (muestra reducida) el logopeda trabaja en colaboración con otros profesionales y para la mayoría el trabajo con la familia es fundamental ( $96 \%$ y $95,1 \%$ respectivamente).

En cuanto a los campos de actuación aparecen porcentajes elevados para la evaluación, intervención y rehabilitación, siendo menores para prevención e investigación, en ambas muestras y en cuanto a las patologías se observa que la mayoría de encuestados de la M1 presenta mayores porcentajes para todas las patologías mientras que los sujetos de la M2 también responden con porcentajes elevados en, por ejemplo: lectoescritura $(81,2 \%)$, laringectomizados $(81,2 \%)$, ictus $(76,6 \%)$, hipoacusia $(64,1 \%)$, trastorno del espectro autista $(62,5 \%)$ y disfonía $(56,2 \%)$.

Tabla 3.-Distribución de las respuestas dadas a las preguntas 22 a la 29.

\begin{tabular}{|c|c|c|c|c|}
\hline \multirow[b]{2}{*}{ 22. El logopeda interviene a: } & \multicolumn{2}{|l|}{$\mathrm{N}=299$} & \multicolumn{2}{|c|}{$\mathrm{N}=64$} \\
\hline & $\mathrm{N}^{0}$ de personas & $\%$ & $\begin{array}{l}\mathrm{N}^{0} \text { de } \\
\text { personas }\end{array}$ & $\%$ \\
\hline Bebés & 104 & 34,8 & 17 & 26,6 \\
\hline Niños & 294 & 98,3 & 61 & 95,3 \\
\hline Adolescentes & 264 & 88,3 & 51 & 79,7 \\
\hline Adultos & 253 & 84,6 & 48 & 75 \\
\hline Ancianos & 215 & 71,9 & 33 & 51,6 \\
\hline \multicolumn{5}{|c|}{ 23. Lugar donde un logopeda puede trabajar } \\
\hline Centros de asistencia médica & 163 & 54,5 & 31 & 47,9 \\
\hline Centros de rehabilitación & 247 & 82,6 & 49 & 76,6 \\
\hline Centros Educativos & 247 & 82,6 & 50 & 78,1 \\
\hline Clínica privada & 268 & 89,6 & 50 & 78,1 \\
\hline Asociación & 237 & 79,3 & 44 & 68,8 \\
\hline Centro de atención temprana & 220 & 73,6 & 38 & 59,4 \\
\hline Residencia de ancianos & 199 & 66,6 & 32 & 50 \\
\hline \multicolumn{5}{|l|}{ 24. Cómo trabaja un logopeda } \\
\hline Solo & 28 & 9,4 & 12 & 18,8 \\
\hline Con otros profesionales & 50 & 16,7 & 6 & 9,4 \\
\hline Ambas formas & 221 & 73,9 & 46 & 71,9 \\
\hline \multicolumn{5}{|c|}{$\begin{array}{l}\text { 25. Es importante que el logopeda trabaje junto a } \\
\text { la familia }\end{array}$} \\
\hline Sí & 287 & 96 & 61 & 95,3 \\
\hline No & 12 & 4 & 3 & 4,7 \\
\hline \multicolumn{5}{|c|}{ 26. Campo de actuación de un logopeda } \\
\hline Intervención & 239 & 79 & 47 & 73,4 \\
\hline
\end{tabular}




\begin{tabular}{lcccc}
\hline Evaluación y diagnóstico & 232 & 77,6 & 52 & 81,3 \\
Rehabilitación & 280 & 93,5 & 56 & 87,5 \\
Prevención & 178 & 59,5 & 26 & 40,6 \\
Investigación & 163 & 54,4 & 27 & 42,2 \\
\hline 27. El logopeda interviene a & & & & \\
Personas que han sufrido un ictus & 248 & 82,9 & 49 & 76,6 \\
Personas con Trastorno de Espectro del Autismo & 212 & 70,9 & 40 & 62,5 \\
Personas con deterioro cognitivo & 172 & 57,5 & 29 & 45,3 \\
Personas con dolores de espalda por postura & 8 & 2,7 & 2 & 3,1 \\
Personas con disfonía & 242 & 80,9 & 36 & 56,2 \\
Personas con dificultades en la lectura ylo escritura & 245 & 81,9 & 52 & 81,2 \\
Personas con helomas & 17 & 5,7 & 5 & 7,8 \\
Personas con una laringectomía & 256 & 85,6 & 52 & 81,2 \\
Personas con deformidades en los pies & 5 & 1,7 & 1 & 1,6 \\
Personas con pie diabético & 9 & 3 & 2 & 3,1 \\
Personas con dificultades en la deglución & 163 & 54,5 & 19 & 29,7 \\
Personas con hipoacusia & 209 & 69,9 & 41 & 64,1 \\
Personas con labio leporino & 189 & 63,2 & 34 & 53,1 \\
\hline 28. A quien es más probable que trate & un & & & \\
logopeda & & & & \\
Locutor de radio & 285 & 95,3 & 61 & 95,3 \\
Profesor & 239 & 79,9 & 49 & 76,6 \\
Ciclista & 14 & 4,7 & 4 & 6,3 \\
Bailarín & 14 & 4,7 & 4 & 6,3 \\
Senderista & 14 & 4,7 & 4 & 6,3 \\
\hline 29. Un logopeda ofrece SAAC & & & & \\
Sí & 253 & 84,6 & 52 & 81,3 \\
No & 46 & 15,4 & 12 & 18,7 \\
\hline Fuente: elaboracion propla
\end{tabular}

Fuente: elaboración propia.

\section{3.-Valoración de la profesión.}

Los resultados sobre la valoración de la profesión aparecen recogidos en la siguiente tabla (ver Tabla 4). Además, se hizo una pregunta abierta del porque creían que el logopeda era necesario.

En general, resaltar de esta Tabla 4 como los porcentajes entre M1 y M2 son similares (98,3\% y $96,9 \%$, respectivamente) ante la pregunta de si el papel del logopeda es necesario. El $94 \%$ y $90,6 \%$, respectivamente consideran que la labor del logopeda no la puede desarrollar otro profesional. Si fuera necesario acudirían al logopeda (97\% y $96,9 \%$, respectivamente). $Y$ hay un desconocimiento de la logopedia ( $63,2 \%$ y $62,5 \%$, respectivamente).

Tabla 4.-Distribución de las respuestas dadas a las preguntas 30 a la 33.

\begin{tabular}{lcccc}
\hline & \multicolumn{2}{c}{$\mathbf{N = 2 9 9}$} & \multicolumn{2}{c}{$\mathbf{N}=\mathbf{6 4}$} \\
\hline 30. El servicio de logopedia es conocido & $\begin{array}{c}\mathbf{N}^{\mathbf{0}} \mathbf{d e} \\
\text { personas }\end{array}$ & $\%$ & $\begin{array}{c}\mathbf{N}^{0} \text { de } \\
\text { personas }\end{array}$ & $\%$ \\
Sí & 110 & 36,8 & 24 & 37,5 \\
No & 189 & 63,2 & 40 & 62,5 \\
31. El papel del logopeda es necesario & & & & \\
Sí & 294 & 98,3 & 62 & 96,9 \\
No & 5 & 1,7 & 2 & 3,1 \\
32. La función del logopeda la puede hacer otro & & & & \\
professional & 18 & 6 & 6 & 9,4 \\
Sí & & & & \\
\hline
\end{tabular}




\begin{tabular}{lcccc}
\hline No & 281 & 94 & 58 & 90,6 \\
33. Si fuese necesario, acudiría al logopeda & & & & \\
Sí & 290 & 97 & 62 & 96,9 \\
No & 9 & 3 & 2 & 3,1 \\
\hline
\end{tabular}

Fuente: elaboración propia.

\section{4.-Discusión.}

En general, nuestros resultados ponen de manifiesto en cuanto a las variables sociodemográficas que hay una mayor presencia de mujeres que de hombres en ambas muestras. También, existe un número elevado de participantes procedentes de Castilla- La Mancha, esto se debe a que la mayoría de las personas a las que se les realizó el cuestionario son de dicha Comunidad Autónoma. Existe un gran número de participantes de edades en torno a los 20 años y en torno a los 50 . Esto puede deberse a que un alto porcentaje de las personas a las que se les envió este cuestionario eran jóvenes estudiantes; por otro lado, en España la población más numerosa es de una edad aproximada a los 50 años. Sin embargo, la muestra es escasa en personas mayores de 70 años, esto puede deberse a que esta encuesta se pasó por soporte digital donde las personas mayores tienen falta de recursos y conocimiento con respecto a los medios digitales.

Con respecto al contacto que han tenido los participantes con la logopedia si analizamos los resultados de la muestra reducida una amplia mayoría dice saber lo que es un logopeda y que Logopedia es un Grado Universitario, siendo la patología más frecuente por la que se consulta al logopeda las dificultades en el habla (dislalia y disfemia), siendo estas las más habituales en edad infantil y por dificultades en la voz (fatiga vocal, sensación de esfuerzo, dolor en la laringe, nódulos, disfonías, etc.

En cuanto al coste de las sesiones, los resultados ponen de manifiesto desconocimiento del precio. Este resultado puede deberse a que el precio varía en función del lugar de residencia, el nivel económico de la población y su demanda, si bien existe una tarifa promedio regulada (Colegio de Logopedas de la Comunidad Valenciana, 2016). Sin embargo, la mayoría de los participantes (incluso de la muestra reducida) saben que sí pueden acceder a este servicio a través de la Seguridad Social. Aunque este dato sea positivo, puede resultar curioso, ya que la figura del logopeda es escasa en las organizaciones públicas como por ejemplo los centros hospitalarios lo que produce largas listas de espera y muchos pacientes solicitan tratamiento en un centro logopédico privado. Por tanto, sería necesario un mayor número de logopedas en los centros sanitarios públicos y una mayor visibilidad de la logopedia en la Seguridad Social, para que así toda persona que lo necesite pueda acceder al logopeda de manera gratuita.

A la pregunta si alguna vez han recibido información acerca de la logopedia, se observa cómo si se ha recibido información lo que podría significar un aspecto favorable ya que la logopedia se da a conocer a través de diferentes formatos. Uno de los medios por los que la mayoría ha recibido información han sido las redes sociales y a través de la opción de respuesta "otros medios". Desconocemos que puede indicar esa categoría, si bien se podría pensar, que dentro de la categoría "otros medios", se encuentra el "boca a boca" o "boca a oreja", siendo una de las mejores maneras de publicidad. En esta línea, para conseguir que la logopedia 
llegue a un número mayor de la población, los Colegios de Logopedas se encargan todos los años de hacer diferentes congresos por España sobre los aspectos de la logopedia para dar la máxima publicidad y llegar a todo tipo de público (logopedas, personal sanitario, docentes, familiares, etc.), (American Speech-Language-Hearing Association, 2016; Pimentel et al., 2010).

En cuanto al campo de actuación de un logopeda y las alteraciones que trata, al igual que otros autores (Breadner et al., 1987; Mahmoud et al., 2014) los porcentajes son más elevados para el colectivo de niños, adolescentes y adultos frente a bebés y ancianos. Sin embargo, es interesante destacar que la intervención logopédica en bebés, aunque puede ser un campo de actuación poco conocido es posible (Guijarro, Martínez-Lorca, González, Zabala-Baños y Martínez-Lorca, 2016). Igualmente, la intervención logopédica también tiene lugar en personas mayores.

Respecto al lugar donde un logopeda trabaja, los mayores porcentajes, en ambas muestras se dan en clínicas privadas. Efectivamente, muchos logopedas ejercen su profesión en clínicas privadas como pone de manifiesto la Encuesta sobre la Situación Actual de la Profesión de Logopedia (Colegio de Logopedas de la Comunidad Valenciana, 2016). Sin embargo, es llamativo como hallamos elevados porcentajes para los centros educativos. La figura del logopeda no suele estar presente en los Centros Educativos Ordinarios Públicos. Por tanto, este resultado, podría deberse a la confusión existente entre el logopeda y el maestro de audición y lenguaje tal y como sugieren Breadner et al. (1987) y Mahmoud et al. (2014).

A la pregunta si el logopeda trabaja en solitario, junto a otros profesionales o de ambas formas, nuestros datos refutan la presencia del trabajo multidisciplinar, así como la importancia del trabajo con la familia. Por lo que respecta a los campos de actuación del logopeda nuestros resultados reflejan como los campos mayoritarios son la evaluación y diagnóstico, intervención y rehabilitación por ser los campos tradicionales (Acosta, 2005). Sin embargo, en nuestro estudio aparecen porcentajes bajos para la prevención y la investigación. En el futuro se debe fomentar entre los profesionales de la logopedia el trabajo preventivo por ser fundamental para evitar o retrasar la aparición de dificultades, alteraciones, patologías, etc., así como la investigación para que esta disciplina crezca, amplié los campos de actuación e innove.

Otra de las preguntas se refería a qué profesionales puede tratar un logopeda destacando porcentajes muy similares entre las dos muestras para los locutores de radio y profesores. Por tanto, podríamos decir que nuestros datos reflejan que hay un conocimiento de la logopedia por parte de los sujetos del estudio, pues identifican a esas dos profesiones como usuarios más frecuentes en el tratamiento logopédico.

Por último, con respecto a la valoración de la profesión, al igual que otros trabajos (Breader et al., 1987; Pimentel et al., 2010; Santo et al., 2016; Shin Ying Chu et al., 2019) nuestros resultados manifiestan como el papel del logopeda es necesario tal y como sugieren los sujetos al explicitar en la pregunta abierta alguna de las siguientes respuestas "porque ayuda a otras personas a comunicarse mejor", "mejora la calidad de vida de los pacientes", "es 
necesario al igual que otros profesionales como un oftalmólogo, un fisioterapeuta o un dentista", disminuye las dificultades del habla de los niños" y "porque ayuda a aquellas personas que por motivos profesionales, circunstanciales, anatómicos o genéticos tienen algún tipo de dificultad". Además, hallamos como la función del logopeda no la puede hacer otro profesional y si fuera necesario la mayoría de los participantes acudirían al logopeda. Por tanto, podríamos decir que hay una valoración del logopeda, se le reconoce como un profesional cualificado y las intervenciones logopédicas pueden lograr la mejoría en caso de necesitar la profesionalidad de un logopeda. Sin embargo, a pesar de estos resultados, sorprende como ambas muestran opinan que todavía hay muchas personas que no conocen el servicio de la logopedia.

Entre las limitaciones de la presente investigación, en primer lugar, el tamaño de la muestra y la localización geográfica de la misma puede considerarse reducida y poco representativa a fin de poder realizar generalizaciones. Para un estudio más exhaustivo, sería necesario un mayor número de participantes de distintas edades y comunidades autónomas, pues en el presente trabajo hay algunas que no están representadas. En segundo lugar, el cuestionario utilizado ha sido elaborado "ad hoc" con la falta de validez y fiabilidad del mismo, por lo que también dificulta la generalización de los resultados. Finalmente, la existencia de poca investigación y por tanto de poca evidencia en este campo del conocimiento de la logopedia en España, abre una importante puerta a nuevos proyectos de investigación, pudiendo suponer una importante área de mejora para dar a conocer de una manera más clara y concisa la logopedia.

\section{5- Conclusión.}

Podemos decir que en cuanto al contacto que tienen los participantes con la logopedia, los sujetos conocen la existencia de la logopedia, aunque se da un desconocimiento de la tarifa profesional. También, un alto porcentaje de los participantes recibieron información sobre la logopedia a través de diferentes medios de difusión, si bien, debería haber una mayor difusión publicitaria, pues, como hemos visto, no todos los sujetos sabían que se podía acceder a la logopedia a través de la Seguridad Social. En cuanto a este último aspecto, sería necesario aumentar desde las administraciones públicas el número de logopedas en los centros hospitalarios públicos, para que todas aquellas personas que lo necesiten sean atendidas.

Por lo que respecta al campo de actuación de un logopeda y las alteraciones, dificultades y trastornos que trata, parece que existe un desconocimiento respecto al trabajo con bebés y personas mayores. Igualmente, se desconoce las funciones de prevención e investigación. También hay confusión con el maestro de audición y lenguaje.

En cuanto a la valoración del logopeda, encontramos que los sujetos consideran que el logopeda es necesario, es un profesional ampliamente cualificado y si fuera necesario acudirían al logopeda, sin embargo, este dato positivo se ve empañado por el hecho de que el servicio de logopedia no es conocido para un porcentaje considerable de nuestros sujetos.

Por tanto, la hipótesis que se planteó al comienzo del estudio se ha cumplido, pues existe conocimiento de la logopedia, aunque exista desinformación sobre algunos aspectos, como 
ocurre en otros estudios (Breadner, 1985; Mahmoud et al., 2014; Santo et al., 2016; Shin Ying Chu et al., 2019). Por tanto, se necesitan realizar futuras investigaciones a fin de determinar el grado de conocimiento de la logopedia por la población en general.

\section{6.-Referencias.}

Acosta, V. (2005). Evaluación, intervención e investigación en las dificultades del lenguaje en contextos inclusivos. Revisión, resultados y propuestas. Revista de Logopedia, Foniatría y Audiología, 25(4), 148-161. doi: 10.1016/S0214-4603(05)75833-2

American Speech-Language-Hearing Association (ASHA). (2016). Scope of practice in speech-language pathology [Scope of practice]. Retrieved from https://www.asha.org/policy/SP2016-00343/

Breadner, B., Warr-Leeper, G. \& Husband, S. (1987). A study of public awareness of speechlanguage pathology: Then and now. Age (Years), 18(19), 10-13.

Colegio Oficial de Logopedas de la Comunidad Valenciana. (2016). Encuesta sobre la situación actual de la profesión de logopedia. Recuperado de: http://www.colegiologopedascv.org/sites/default/files/INFORME_COLCV_2016.pdf

De Salas, M.I. (2009). La publicidad en las redes sociales: de los invasivo a lo consentido. Revista Icono 14(15), 75-84.

Guijarro, C., Martínez-Lorca, M., González, J., Zabala-Baños, M., y Martínez-Lorca, A. (2016). Conocimiento de la estimulación prenatal en los programas de educación maternal en Atención Primaria por parte de las mujeres gestantes. Revista Clínica de Medicina de Familia, 9(2), 83-90.

López, T. (2017). Un 30\% de la población necesitará los servicios de un logopeda en algún momento de su vida. València Extra. Recuperado de: https://valenciaextra.com/es/beneficios-logopedia/

Pimentel, A.G.L., Lopes Herrera, S.A., y Duarte, T.F. (2010). Conhecimento que acompanhantes de pacientes de uma clínica-escola de Fonoaudiologia tem sobre a atuação fonoaudiológica Knowledge persons accompanying patients in a SpeechLanguage Pathology school-clinic have about the work of. Rev Soc Bras Fonoaudiol, 15(1), 40-6. Doi: 10.1590/S1516-80342010000100009.

Santo, C., Franco, E., Arakawa, A., Xavier, A., Bastos, J., y Caldana, M. (2016). Conhecimento sobre a atuação fonoaudiológica em um município da região amazônica. Distúrbios da Comunicação, 28(1), 142-150.

Shin Ying Chu., Keng Ping Tang., Grace McConnell, Hanif Farhan Mohd Rasdi y Man-Ching Yuen. (2019). Public perspectives on communication disorders and profession of 
Revista Internacional de Apoyo a la Inclusión, Logopedia, Sociedad y Multiculturalidad. Volumen 6, Número 2, Junio 2020, ISSN: 2387-0907. DOI: https://doi.org/10.17561/riai.v6.n2.1

speech-language pathology. Speech, Language and Hearing, 22(3), 172-182. doi: https://doi.org/10.1080/2050571X.2019.1570705 